\title{
call for papers
}

JOM is seeking contributions on the following topics for 2021. For the full Editorial Calendar, along with author instructions, visit www.tms.org/EditorialCalendar.

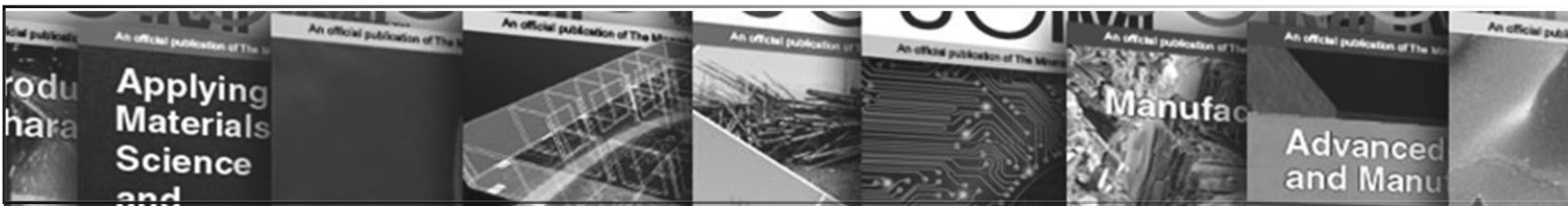

October 2021

Manuscript Deadline: May 1, 2021

Topic: Corrosion in Heavy Liquid Metals for Energy Systems

Scope: This topic invites papers on studies related to heavy liquid metal (HLM) such as $\mathrm{Pb}$ and lead bismuth eutectic compatibility with structural materials including corrosion and liquid metal embrittlement. In addition, technological aspects of HLM technology including chemistry control methods, filtering, in-situ characterization techniques, forced and natural convection methods, and flow measurements are also included in this topic.

Editors: Osman Anderoglu, Alessandro Marino, and Peter Hosemann

Sponsors: Corrosion and Environmental Effects Committee and Nuclear Materials Committee

\section{Topic: Informatics-Enabled Design of Structural Materials}

Scope: Informatics-enabled design is a paradigm shift for materials engineering and has led to many breakthroughs within the last decade. For structural materials, an array of challenges persist due to the need for quantitative evaluation of competing performance metrics across many time and length-scales. This special topic aims at capturing the needs and limitations of informatics toolsets for design of structural materials. We invite articles that highlight recent advances and set the scope for future.

Editors: Jennifer L.W. Carter and Amit K. Verma Sponsor: Mechanical Behavior of Materials Committee

Topic: Materials for Small Nuclear Reactors and Micro Reactors, including Space Reactors

Scope: Small nuclear reactors, including micro-reactors, small modular reactors, space reactors, and off-grid reactors rely on different materials and manufacturing processes than those in large-scale power plants: molten salts as coolants and fuels, heat-pipes for heat removal, metal hydrides as high-temperature moderators, fuels for higher burnup and accident tolerance, etc. They also require novel structural materials and understanding of material interactions. This special topic focuses on materials research and experimental and modeling/ simulation for small nuclear reactors.

Editors: Sven C. Vogel, Raluca O. Scarlat, Aditya P. Shivprasad, and Marisa Monreal

Sponsor: Nuclear Materials Committee

\section{November 2021 Manuscript Deadline: June 1, 2021} Topic: Advanced High-Strength Steels Scope: Advanced high-strength steels (AHSS) have been widely used in commercial vehicles for decades. New AHSS are being actively researched in academia and industry. This special topic focuses on the latest developments in AHSS, including high-strength lowalloy (HSLA), dual-phase (DP), transformation-induced plasticity (TRIP), complex phase (CP), martensitic, quenched \& partitioned (Q\&P), medium manganese, TRIPassisted bainitic ferrite (TBF), press-hardened steel (PHS), twinning-induced plasticity (TWIP), and low density steels. Editors: M.X. Huang and Ana Araujo Sponsor: Steels Committee

\section{Topic: Advances in Multi-modal Characterization of Structural Materials}

Scope: Progress in the development of instrumentation and workflows that enable the collection of various data modalities have provided novel insights into material behavior. This special topic will focus on the application of varied characterization approaches in both $2 \mathrm{D}$ and $3 \mathrm{D}$, across multiple length scales and/or imaging modalities, for structural materials. Papers that focus on the development and application of advanced segmentation and data fusion approaches for quantitative data analysis are also invited.

Editors: Andrew T. Polonsky and Amit Pandey Sponsor: Advanced Characterization, Testing, and Simulation Committee 


\section{Topic: Latest Developments in Manufacturing and Recycling of Refractory Materials}

Scope: Renowned for their unique properties, refractory materials have widespread applications in electronic, nuclear, and defense industries. Although powder metallurgy is still the only route for major commercial production, manufacturing and recycling technologies have made great strides in processing of refractory materials. The focus of this special topic includes recent advances in overcoming process challenges or improving material performances. Manuscripts covering the latest experimental and theoretical studies especially focusing on recycling of refractory metals are invited.

Editors: Chai Ren and Ravi Enneti

Sponsor: Refractory Metals and Materials Committee

\section{Topic: Silicon Technologies}

Scope: This topic covers silicon production from quartz and carbon to the most important feedstock for crystalline solar cells. Characterization of single-crystalline silicon, silicon defects, and behavior of impurities are included, as well as the use of silicon as a storage material, and use of silicon melt to store and generate energy. Recycling of silicon compounds, solar cells, electronic components, and life-cycle of silicon-related technologies are also covered. Editors: Shadia Ikhmayies

Sponsors: Recycling and Environmental Technologies Committee and Materials Characterization Committee

\section{December 2021 Manuscript Deadline: July 1, 2021 Topic: Advanced Casting and Melt Processing Technology for Light Alloys}

Scope: This topic covers the newly developed or significantly improved casting and melt processing technologies applicable to light alloys. This may include advanced studies on the improvement of structure; optimization of phase composition, mitigation of casting defects as well as advances in casting and melt treatment technology. Also considered is the extension of the technology to recycled alloys. Both experimental and modelling studies will be considered, the latter requiring experimental validation.

Editor: Dmitry Eskin

Sponsor: Aluminum Committee
Topic: Advances in Processing, Manufacturing, and Applications of Magnetic Materials

Scope: We welcome submission of papers on advances for synthesizing, processing, and characterization of magnetic materials including permanent and soft magnets, energy conversion, and multiferroic materials (such as magnetocaloric, magnetoelastic, magnetoelectric and magnetoresistive materials). Applications of interest include biological applications of magnetism, sensors and actuators, energy harvesting, motor-generators, transformers and inductors, and memory applications. Work on discovery, advanced manufacturing, processing and characterization techniques applied to the relevant magnetic materials and their applications, is strongly encouraged.

Editors: Scott McCall and Ikenna Nlebedim

Sponsors: Magnetic Materials Committee

\section{Topic: Corrosion and Protection of Materials at High Temperatures}

Scope: Papers on all aspects of high-temperature corrosion and protection of materials are invited. Examples of topics include oxidation in different atmospheres, molten salt corrosion, metal dusting, halogen attack, etc. Papers dealing with surface modification for high-temperature corrosion protection are also invited.

Editors: Vilupanur Ravi and Ramprashad Prabhakaran

Sponsor: Corrosion and Environmental Effects Committee

\section{Topic: Surface Engineering for Improved Corrosion or Wear Resistance}

Scope: Corrosion and wear are surface phenomena and therefore, surface engineering has been used to improve both properties. Coatings, surface alloying, gradient structures, nanocrystallization, and inhibitors have been applied to tailor the surfaces for improved corrosion and wear resistance. This special topic focuses on capturing recent advancements in: 1) surface engineering technologies to improve corrosion and/or wear resistance and 2) theoretical understanding of corrosion and/or wear behavior of the surfaces.

Editors: Tushar Borkar, Arif Mubarok, and Rajeev Gupta Sponsor: Surface Engineering Committee

\section{Contribute to JOM}

Visit jom.tms.org to access author tools that will answer your questions during every step of the manuscript preparation process, from determining the appropriate technical topic for your paper to reading the final product on Springerlink.

For further information on contributing to JOM, contact JOM Editor Maureen Byko at mbyko@tms.org.
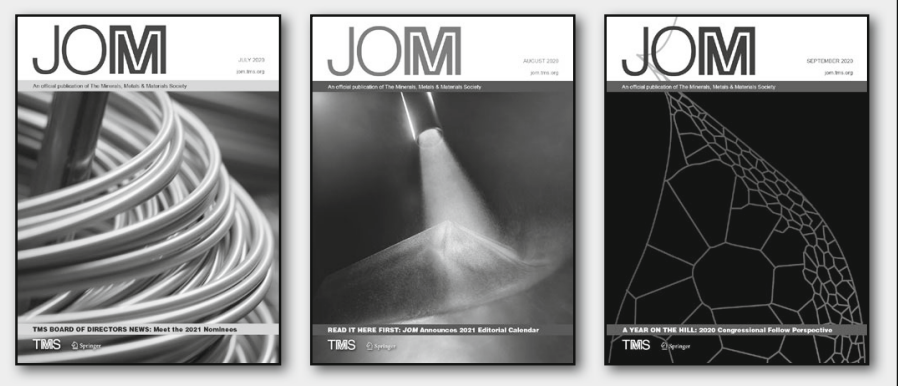